\title{
Sensitivity Analysis of an Automated Formation Flight Based on GPS and Transmission Data Specifications
}

\author{
Mehmet C. Kilic* \\ Innovation Society of Technical University of Braunschweig (iTUBS), Braunschweig, Germany \\ Martin J. Meiboom ${ }^{\dagger}$ \\ German Aerospace Center (DLR), Institute of Flight Systems, Braunschweig, Germany
}

\begin{abstract}
An automated formation flight scenario with one leader and one follower aircraft is considered. In this context, a simulation-based sensitivity analysis is used to investigate how the position keeping performance of the automated formation flight is affected by the specifications of the leader aircraft's flight data that the follower aircraft gathers. The leader aircraft flight data specifications are determined by three parameters: Noise present in the leader flight data, time delay of the data by the time it is acquired by the follower aircraft, and the period at which the follower aircraft gathers the leader flight data. These three parameters are the independent variables of the sensitivity analysis and are varied among consecutive simulations. The sensitivity of the formation flight is assessed by the maximum absolute relative position error during 60 seconds of stable formation flight on a straight and level path. For the sensitivity analysis an example data gathering configuration is used, in which the GPS-based, time-stamped position and velocity measurements of the leader aircraft are sent to the follower through a wireless data link.
\end{abstract}

\section{Nomenclature}

Symbo
$d$
$\boldsymbol{d}_{F}$
$\boldsymbol{e}$
$n$
$\boldsymbol{r}$
$t$
$V$
$\boldsymbol{V}$
$\boldsymbol{X}$
$\overline{\mathbf{x}}$

$\gamma$
$v$
$1 / v$
$\zeta$
$\chi$

$\omega_{n} \quad$ natural frequency, $\mathrm{rad} / \mathrm{s}$

delay, latency, $\mathrm{s}$

position error controller input, $\mathrm{m}$

Sub- and Superscripts

$c \quad$ commanded

$F \quad$ follower aircraft

noise amplitude factor

$L \quad$ leader aircraft

position vector, $\mathrm{m}$

$L F \quad$ follower aircraft relative to leader aircraft

time, $\mathrm{s}$

$r \quad$ position-related

ground velocity, $\mathrm{m} / \mathrm{s}$

ground velocity vector

$x, y, z$ components of a vector

state vector

true and current value of $\mathrm{x},(\mathrm{x}$ : any variable)

Abbreviations

DOF degree-of-freedom

climb angle, rad

GPS global positioning system

transfer rate, sampling rate, $\mathrm{Hz}$

IMU inertial measurement unit

transfer period, $\mathrm{s}$

S\&H sample and hold

damping ratio

course angle (flight path azimuth angle), rad

*Guest Researcher at German Aerospace Center (DLR), Department of Unmanned Aircraft, mehmet.kilic@dlr.de

$\dagger$ Research Scientist at German Aerospace Center (DLR), Department of Unmanned Aircraft, martin.meiboom@dlr.de 


\section{Introduction}

Formation flight is the flight of multiple aircraft coordinated by one leader aircraft, which is a well established capability among manned aircraft. Possible use of a flight in formation is the increase of efficiency due to drag reduction, ${ }^{1}$ the concentration of firepower, ${ }^{2}$ and specialized missions like aerial refueling. ${ }^{3,4}$ As capabilities of unmanned aircraft increase, methods to fly automatically in formation have been extensively studied in the literature concerning flight control, ${ }^{5-11}$ guidance, ${ }^{12-14}$ and coordination of the vehicles. ${ }^{15}$

In order to maintain the formation, the follower aircraft gathers certain information about the leader aircraft. This information may contain flight velocity and relative position, ${ }^{8,11}$ attitude $^{8,10,11}$ and acceleration. ${ }^{11}$ Furthermore, the follower aircraft may gather the information of the leader aircraft either by sensors that it carries on board ${ }^{16}$ or through means of transmission from the leader aircraft. ${ }^{8,10,11}$

The minimum information to perform automated formation flight includes the reception of the 3DPosition from the leader aircraft. ${ }^{3,6,9-11}$ The most frequent means of obtaining position information are pure or IMU-aided GPS ${ }^{3,6,9-11}$ and vision-based techniques. ${ }^{16}$ The accuracy of the formation flight can be increased by using further information of the leader aircraft in the guidance and control algorithms. Translational and rotational velocities, attitude and acceleration information of the leader aircraft are examples for such information. The most frequent methods for obtaining the information is using IMU or navigation solutions combining the IMU and GPS. If the sensors that provide the leader aircraft's information are placed on the leader aircraft, such as GPS and/or IMU, the information is sent to the follower aircraft by a wireless data link. ${ }^{6,9-11,14}$

Regardless of the method of gathering the leader data, this paper focuses on the question how the specifications of acquired leader flight data effect the accuracy of the formation keeping. The aforementioned leader data specifications are defined on a general level in order to cover the common specifications of other possible data acquisition techniques that can be used in a formation flight scenario. In this respect, it is assumed that, regardless of the employed data gathering technique, the leader data will be available to the follower aircraft at specific time periods, with some amount of delay, and error with time-varying amplitude. Hence these three parameters are selected as independent variables for the sensitivity analysis. The parameters are applied to an example data gathering method, in which the follower aircraft collects the GPS-based leader position and velocity data through a wireless data link. A model resembling this set-up is constructed based on ground test data. Filtering the gathered leader data is also considered; this case is represented by a simple dead-reckoning filter. The sensitivity of the formation flight with such a system is then investigated based on simulation-based parameter sweep analysis. The sensitivity analysis results are exemplarily outlined for two particular unmanned aircraft operated by the DLR (German Aerospace Center), which are planned to be used in the flight demonstration of the automated formation flight.

The paper is subdivided into the following sections: A brief introduction to the simulation environment is given in section II, based on Ref. 17. Section III gives a model of gathering the leader aircraft's flight data on the follower aircraft, as well as a possible way of filtering of the leader aircraft data after its acquisition by the follower aircraft. In section IV, the method and the parameter ranges of the simulation-based sensitivity analysis are given, which uses the rigid body simulations and information gathering model presented in sections II and III. Also given in the section IV are the results of the simulation-based sensitivity analysis, summarized in form of graphs. Finally, section V concludes the paper.

\section{Simulation}

\section{A. Simulation Setup}

A hybrid Matlab/Simulink and $\mathrm{C} / \mathrm{C}++$ simulation structure is used. The simulation environment includes the flight dynamical models of the leader and follower aircraft, their guidance algorithms and autopilots, and the model for follower aircraft's acquisition of the leader aircraft's flight data, as depicted in figure 1.

The leader aircraft's flight dynamical model is a 3 DOF point-mass dynamical model. This model is combined with a flight path generator, a path following guidance and feedback-linearization-based autopilot. This set-up enables the leader aircraft to follow a given path closely.

The follower aircraft is modeled by nonlinear 6 DOF equations of motion including aerodynamics, weight and inertia properties, and a propulsion model. It has an already-existing autopilot, which is a cascaded PID controller on the layers of angular rates, attitude angles, velocity components, and $3 \mathrm{D}$ path deviations. The input interface of the controller consists of the commanded velocity components $\left(V_{F c}, \gamma_{F c}, \chi_{F c}\right)$ and path 


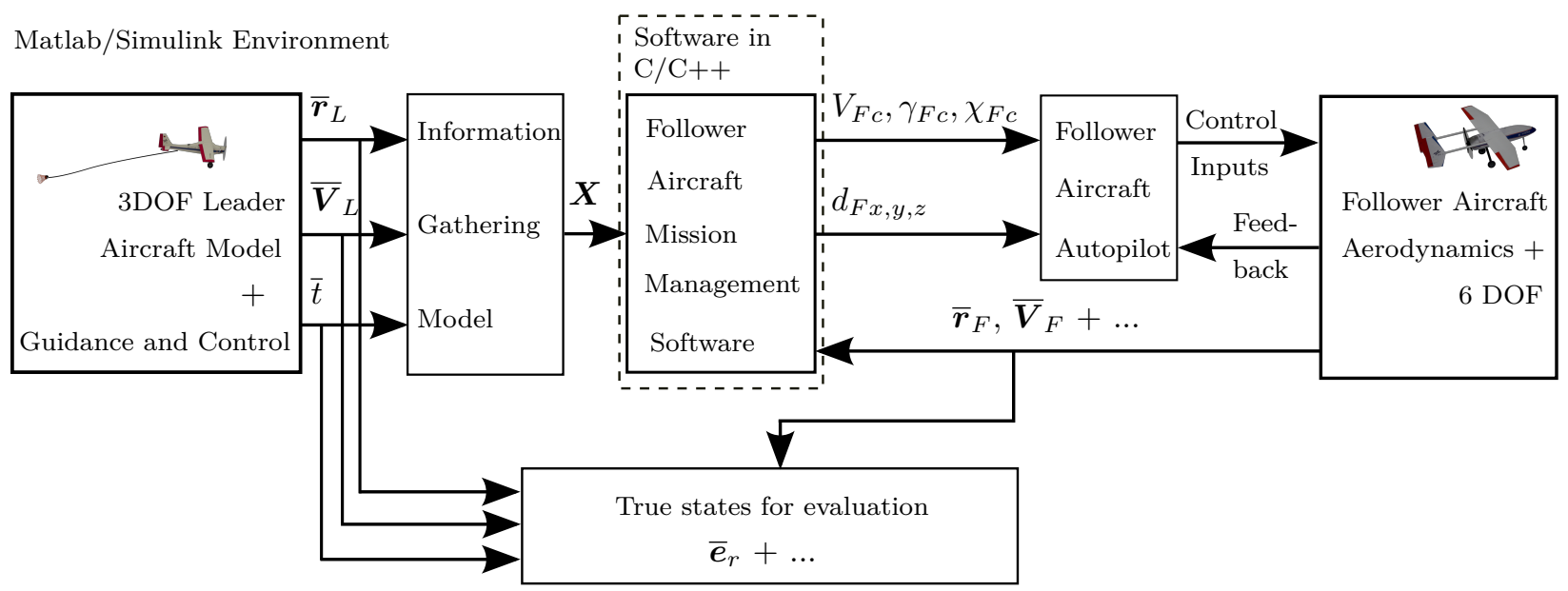

Figure 1. Simulation environment set-up from Ref. 17 (modified)

deviations $\left(d_{F x, y, z}\right)$. The controller is a linear parameter-varying PID controller with second order dynamics on reference signals and feed-forward on some channels. The structure and parameters of the autopilot are kept constant for this work.

The mission manager software of the follower aircraft, which is also used in the real flight tests, is a software written in $\mathrm{C} / \mathrm{C}++$ language. It contains the formation guidance algorithm described below. The reception of the leader aircraft flight data and their possible filtering before using them in the formation guidance algorithm also take place here. The mission management software and all included algorithms are executed at a constant rate of $50 \mathrm{~Hz}$.

The information gathering model simulates the gathered leader aircraft flight data characteristics. The current model is based on collecting the GPS-based, time-stamped position and velocity data of the leader aircraft through a wireless data link. This model is explained in detail in section III.

\section{B. Formation Guidance Algorithm}

The formation guidance algorithm or formation flight controller acts on the relative position error, which is given in equation (1). ${ }^{17}$ It is the difference between the commanded (desired) relative position and current relative position of the follower aircraft with respect to the leader. The components of the relative position error are defined in the leader-fixed reference frame. The leader-fixed reference frame is a right-handed axis system whose $\mathrm{x}$-axis is aligned with the ground velocity of the leader aircraft. The y-axis points to the right of the $\mathrm{x}$-axis and is in the horizontal plane. The z-axis completes the right handed axis system.

$$
\boldsymbol{e}_{r}=\boldsymbol{r}_{L F}-\boldsymbol{r}_{c}=\left[e_{x} e_{y} e_{z}\right] \text {, with } \boldsymbol{r}_{L F}=\boldsymbol{r}_{F}-\boldsymbol{r}_{L}
$$

The x-component of the relative position error, $e_{x}$ is controlled through the $V_{F}$ interface of the autopilot.

$$
V_{F_{c}}=V_{L}+K_{P_{1}} e_{x}+K_{I_{1}} \int e_{x} d t
$$

The guidance algorithm uses the $\chi_{F}$ interface of the autopilot to control the y-component of the error, $e_{y}$ as well as in order to remain at about the same course as the leader.

$$
\chi_{F_{c}}=\chi_{L}+K_{P_{2}} e_{y}
$$

Finally, the z-component of the relative positioning error, $e_{z}$ is handled through the $d_{F z}$ input of the autopilot as shown in equation (4).

$$
d_{F z}=e_{z}
$$

The guidance algorithm's parameters $K$ are tuned for the simulated flight conditions, in which the leader aircraft flies on a straight and level path, with a constant ground speed of $35 \mathrm{~m} / \mathrm{s}$. 
The remaining inputs of the follower autopilot $\left(\gamma_{F c}, d_{F x}, d_{F y}\right)$ are currently not used for formation control.

\section{Follower Aircraft Closed-Loop Dynamics}

The results of the sensitivity analysis will be valid for the particular dynamical system used in the simulations, namely the dynamics of the formation keeping. In order to present the dynamical characteristics of the formation keeping, the closed loop dynamics comprising of mission management, autopilot and follower aircraft is given in terms of step response plots. A unit stepwise change in commanded relative position is used separately along $\mathrm{x}, \mathrm{y}$, and $\mathrm{z}$ components of the formation flight control during the same flight condition that is used for the sensitivity analysis. The dynamical response of relative position components to the stepwise commands are plotted in figure 2. Also given in figure 2 are the natural frequencies and the damping ratios of the second-order transfer function approximations obtained from the given step responses.

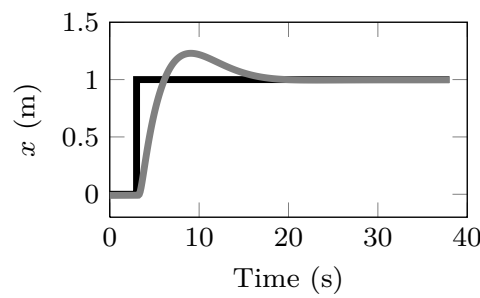

(a)

$$
\begin{aligned}
& \text { Variation of relative x-position. } \\
& \text { Second-order tf. approximation: } \\
& \omega_{n}=0.57 \mathrm{rad} / \mathrm{s} \text {, and } \zeta=0.42
\end{aligned}
$$

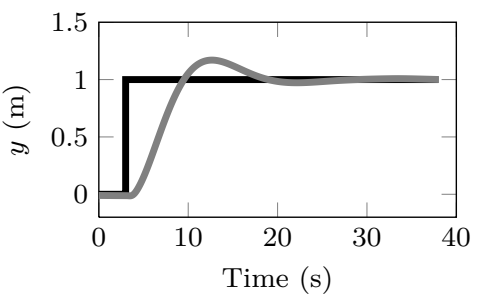

(b)

Variation of relative y-position. Second-order tf. approximation: $\omega_{n}=0.37 \mathrm{rad} / \mathrm{s}$, and $\zeta=0.49$

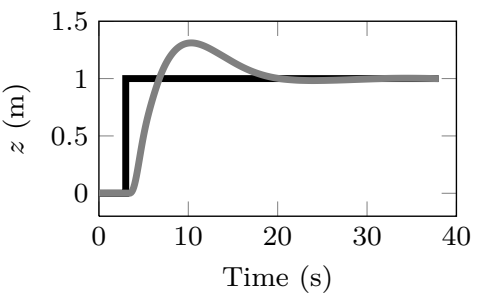

(c)

Variation of relative z-position. Second-order tf. approximation: $\omega_{n}=0.46 \mathrm{rad} / \mathrm{s}$, and $\zeta=0.35$

Figure 2. Follower aircraft relative position responses to unit stepwise changes in the components of commanded relative position. Commanded and actual relative position components are expressed in the leaderfixed reference frame.

\section{Gathering the Leader Aircraft Information on the Follower Aircraft}

Among the different formation flight sensor configurations presented in literature, here a simple sensor configuration is selected for application to the leader and follower aircraft for the planned flight test. In this configuration the leader aircraft uses only its GPS receiver in order to inform the follower aircraft about its state, via a wireless datalink. This readily available sensor configuration is further utilized for the sensitivity analysis as an example case. After an analysis of the GPS and the datalink based on ground tests, a model representing this data acquisition technique is presented below. The data gathering model is also parameterized and these parameters will be the independent parameters of the sensitivity analysis.

\section{A. GPS and Datalink Analysis}

Before reviewing the data link, the GPS receiver is discussed to conclude an error behavior for the formation flight. GPS errors are well known and discussed in the literature. Typical sources of GPS error are listed below.

- Ephemeris data

- Satellite clock

- Ionosphere

- Troposphere

- Mulipath

- Receiver noise

During formation flight, the GPS receivers of both aircraft are close together. Hence several errors will influence both receivers in the same way. The close distance between the GPS receivers result in errors that are similar as in DGPS solutions. The similarity allows us to assume ephemeris and satellite clock errors as zero. ${ }^{18}$ It is also assumed that the attitude of both aircraft are the same during the formation flight on the straight and level path. So it is expected that the GPS receivers of both aircraft will see the same satellite 
constellation. The possibility of the GPS signal blockage due to the leader aircraft is also neglected, as the distance between the two aircraft is sufficiently large compared to their size. Ionosphere and troposphere are partly considered for DGPS, since rover and base station might be separated by multiple kilometers and the satellite signal penetrates spheres with different properties. However, for the planned automated formation flight experiment the maximum distance will be limited to approximately one kilometer, so ionosphere and troposphere errors are negligible as well. The multipath noise might influence the GPS accuracy for large distances between both vehicles through different terrain characteristics during flight. Since this effect declines with decreasing distance, during the formation flight the effects are neglectable. This leaves the uncompensated receiver noise as major error source for both vehicles.

Assuming the receiver noise is not dependent on the amount of velocity, a ground test was designed to verify the accuracy for two relatively positioned, identical GPS receivers. Since the internal kinematic models of a GPS receiver are out of our scope and a static and dynamic test lead to different results, both relative positioned GPS receivers are evaluated in motion. ${ }^{19}$ The test setup consists of two uBlox 6 receivers strapped on a platform one meter apart, configured as an airborne system with $5 \mathrm{~Hz}$ update rate. The motion consisted of circles and straight lines with velocities of 30 to $70 \mathrm{~km} / \mathrm{h}$. A portion of relative position and velocity error results from this ground-test are presented in figure 3. These resulting error values are used as additive noise for simulation, labeled here simply as noise. To later represent the influence of different GPS receivers with higher variance and different update rate the resulting error is parameterized with a multiplicative parameter $n$, as well as a variable transfer period, $1 / v$ in order to vary the transfer rate of the leader information, representing the first two of three used independent variables.

The data link properties are derived from the specifications of two Freewave FGR2 $900 \mathrm{MHz}$ Modems. The data sheet provides information about the maximum delay of $21 \mathrm{~ms}$ considering no data loss. However taking data loss into account the latency of packages can increase significantly. Thus the latency between both modems was determined through an echo test. The message length and update rate were represented by dummy data similar to the original GPS protocol. During the test the antenna orientation and position were varied. The test revealed a worst case one way message latency of approximately $75 \mathrm{~ms}$ for a distance of $40 \mathrm{~m}$ on ground. During flight test the latency might decrease due to closer distance and less disturbances. Since the data link test did not show a fixed latency, the latency of the received position and velocity data will be used as the third independent variable, $d$. Note, that time varying latencies (Jitter) are not considered.

\section{B. Data Gathering Model for the Formation Flight}

The data gathering model simulates the collection of timestamped position and velocity data of the leader aircraft onboard the follower aircraft. The sensor and data link model is shown in figure 4 . It takes the characteristics of both relatively positioned GPS receiver and the data link into account.

The noise data shown in figure 3 is multiplied by $n$, before it is added to the components of the leader absolute position vector $\boldsymbol{r}_{L}$, whose components are expressed in North-East-Down axis system. The dimensionless real number $n$, which is called the noise amplitude factor, therefore determines the amplitude of the noise which is added on the leader position and velocity data. The vertical position component of the noise data is added on the z-component of the leader aircraft position $\boldsymbol{r}_{L}$. The horizontal position component of the noise data is divided equally for the North and East components and added on the corresponding components of $\boldsymbol{r}_{L}$. The noise on the horizontal speed and the course angle are added on the ground speed and course angle of the leader aircraft.

The S\&H (Sample \& Hold) block simulates the update rate of the GPS receiver. It is determined by the sampling frequency $v$. The inverse of this number, namely $1 / v$ is used in this paper and is called the transfer period. The transfer period of leader data is the constant time interval between two consecutive leader data sets that the follower aircraft receives. This parameter allows us to take into account GPS receivers with higher or lower update rates, allowing the follower aircraft to receive more or less updates from the leader aircraft per unit time. The transfer delay $d$ of the leader aircraft's data is the time interval, by which the leader data lags behind its current values, by the time it is received by the follower aircraft. The parameter range represents all the latencies that are caused by the GPS and wireless data link system, also including the scenarios like package loss.

Through the model described above, the leader data are received by the follower aircraft mission management software.

The presented data gathering method is one example case from other sensor possibilities presented in the literature. However, with the parameters of the model, characteristics of other sensors with different 

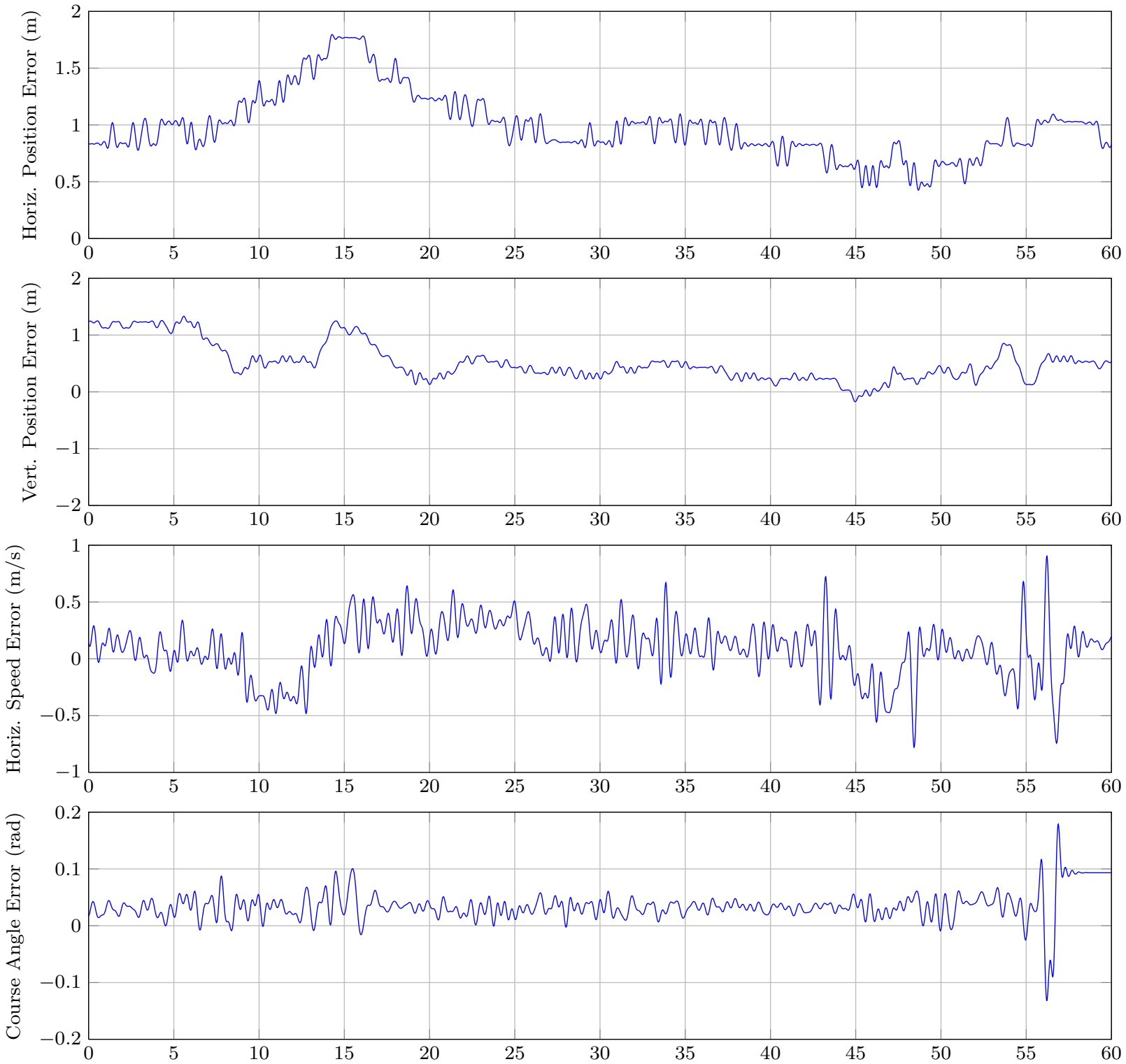

Figure 3. Varying relative position (horizontal and vertical) and velocity error (i.e. noise) obtained from ground tests using GPS. The standard deviations of the data components are from top to bottom graphs: 0.28 $\mathrm{m}, 0.33 \mathrm{~m}, 0.24 \mathrm{~m} / \mathrm{s}$ and $0.025 \mathrm{rad}$. The figure shows only the portion of the ground test data, which is used in the formation flight simulations of 60 -second duration.

accuracy, update rate and latency properties can also be represented. By this, the results of the presented sensitivity analysis allow drawing conclusions also for other types of sensor configurations.

\section{Leader Prediction Algorithm}

In a real-life application it is likely that the gathered leader data are processed by a predicting filter before their usage in the guidance algorithms. The filter can be used for synchronizing the data of both aircraft, increasing the rate of update of the data, and compensate for the delays, as presented by Ref. 20. In order to also represent this case, a second configuration of the application of this data gathering technique is also included. In this configuration, a dead reckoning algorithm processes the leader data acquired by the follower aircraft, before using them in the guidance and control algorithms. It uses the leader's velocity information for compensating delays and providing estimations of the position data also during the instants in which no leader data is received. 


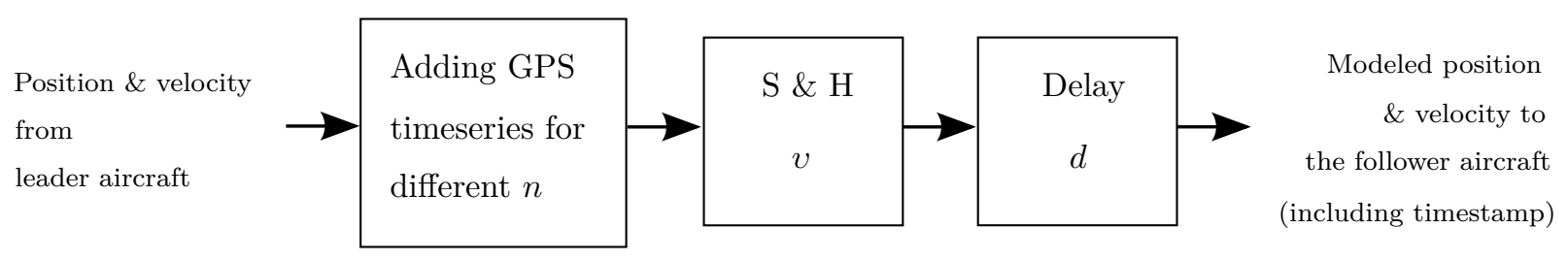

Figure 4. FFM for gathering GPS data through a wireless data link

The leader prediction algorithm represents a simple filter to pre-process the leader aircraft flight data, after their acquisition on the follower mission manager, before using them in the formation flight guidance algorithms. It executes in the mission management software, depicted in figure 1. The leader aircraft's information arrives to the follower aircraft's prediction algorithm as a package, which is shown in equation (5) as a vector. The data package contains the position of the leader aircraft in the North-East-Down reference frame, its ground velocity components in polar coordinates, and a time stamp to which the data belongs.

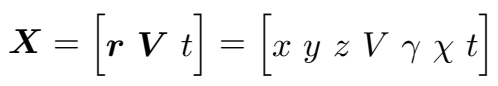

The algorithm predicts the leader aircraft's position in the North-East plane and its course angle using a first order dead-reckoning approach, as shown in algorithm 1. Since the leader aircraft is commanded to fly level and at a constant ground speed, prediction of velocity, climb angle and down position are not included in the prediction algorithm. They are assumed to remain constant through the flight. The prediction algorithm also requires that, the leader and follower aircraft have the same time base, which could be obtained by synchronizing the time of both aircraft with the GPS timebase.

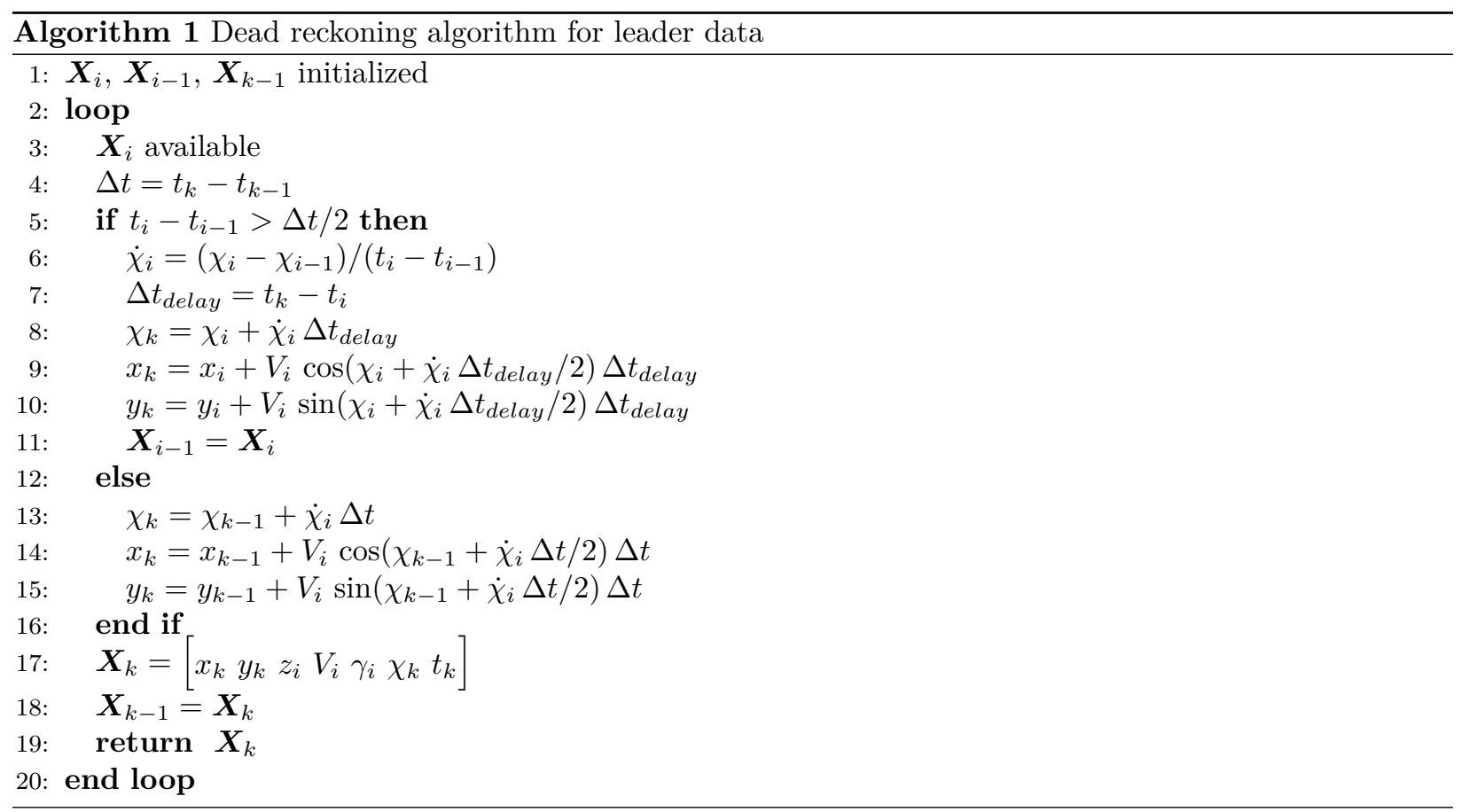

The index $k$ shows the current iteration count of the algorithm, which runs recursively within the mission management software of the follower aircraft. The variable $t_{k}$ denotes the time stamp at the current iteration, and it is available to the algorithm whenever required. The index $i$ belongs to the most recently-received leader data package. The input of the algorithm is $\boldsymbol{X}_{i}$ which holds the most recently-received leader data until new leader data is received. The values assigned to the contents of $\boldsymbol{X}_{i}$ do not necessarily change at each iteration of the prediction algorithm. In the pseudo code notation of algorithm 1, any operation acting on $\boldsymbol{X}$ also applies to all the information that it contains. The condition $t_{i}-t_{i-1}>\Delta t / 2$ checks if new leader 
data is received. It relies on the assumption that, the time difference between the two most recent leader data cannot be smaller than half the processing period $\Delta t / 2$ of the algorithm.

The lines 5-11 of algorithm 1 is the delay compensation component. The state prediction part of algorithm 1 is the lines 12-15, which propagates the leader states forward. Assuming that the leader data transfer period $t_{i}-t_{i-1}$ and the algorithm's execution period $\Delta t$ are constant, the prediction component executes about $\left(t_{i}-t_{i-1}\right) / \Delta t-1$ times at each transfer period. Whereas the delay compensation takes place once at each transfer period.

\section{Formation Flight Sensitivity Analysis}

The sensitivity analysis is based on consecutive simulations, making use of the simulation environment described above. The simulation is run repetitively, varying the three independent variables at each simulation run. These are the parameters, which are applied to the example data gathering configuration of GPS and data link system. The ranges of independent variables and the conditions under which the simulations are performed are summarized in Table 1 .

\section{Flight Condition}

Leader aircraft ground speed, $V_{L}$ :

$35 \mathrm{~m} / \mathrm{s}$, constant

Leader aircraft flight path:

Wind and turbulence:

Straight and level path at $1450 \mathrm{~m}$ altitude

Disabled

\section{Independent Variables}

Leader flight data noise amplitude factor, $n: \quad 0,0.5,1,1.5,2$

Leader flight data transfer delay, $d: \quad 0,0.01,0.02,0.04,0.08,0.16,0.2$ seconds

Leader flight data transfer period, $1 / v: \quad 0.02,0.05,0.1,0.2,0.5,1$ seconds

Other

Follower data processing period:

0.02 seconds

Table 1. Simulation conditions and independent variables

In the table, the follower aircraft's data processing period represents the time interval between the two consecutive executions of the formation flight algorithms. In the simulations, the leader aircraft flies on a straight and level path at the altitude of 1450 meters. The simulations are started in a flight condition, in which the leader and the follower aircraft are outside the formation configuration. The simulations are set to run long enough to give the follower aircraft enough time to establish a stable formation flight. After the formation flight is stabilized, the true relative position error, $\overline{\boldsymbol{e}}_{r}$ during 60 seconds of formation flight is taken from the simulation environment for analysis. The term $\overline{\boldsymbol{e}}_{r}$ is defined by equation (6), which is calculated with true position information taken directly from the flight dynamics models, as shown in figure 1.

$$
\overline{\boldsymbol{e}}_{r}=\overline{\boldsymbol{r}}_{L F}-\boldsymbol{r}_{c}=\left[\bar{e}_{x} \bar{e}_{y} \bar{e}_{z}\right]
$$

The true relative position error during 60 seconds of formation flight is recorded with respect to time. For the applications of the formation flight, such as aerial refueling, the maximum of the error during the concerned time is more critical. Therefore, for the analysis of the simulation data magnitudes of the components of the maximum relative position error $\left|\overline{\boldsymbol{e}}_{r}\right|_{\text {max }}$, namely $\left|\bar{e}_{x}\right|_{\text {max }},\left|\bar{e}_{y}\right|_{\text {max }}$, and $\left|\bar{e}_{z}\right|_{\text {max }}$ are used, for which the $\mathrm{x}$-component is given in equation (7). The $\mathrm{y}$ and $\mathrm{z}$ components can be written in the same fashion. The error components are expanded on the axes of the leader-fixed reference frame.

$$
\left|\bar{e}_{x}\right|_{\max }=\max \left(\left\{\left|\bar{e}_{x}\right|(t): t=t_{\text {start }}, \ldots, t=t_{\text {end }}\right\}\right), \quad\left|\bar{e}_{x}\right|(t)=\sqrt{\bar{e}_{x}^{2}(t)}
$$

Two sets of simulations are run in which the given parameter range are used. In the first set, the gathered leader aircraft data is directly used in the guidance and control algorithms. This means that the guidance and control algorithms process the same, the most recent available leader aircraft flight data until the new data of the leader is received. In the second set of simulations, the gathered leader data are first processed by the dead-reckoning-based prediction algorithm and then used in the guidance and control algorithms. 
Among these simulation runs, the independent variables shown in the table are the only variables, which vary from simulation to simulation. All other parameters and the simulation structure remain unchanged. This type of sensitivity analysis, in which only one independent variable is varied for each simulation run corresponds to the one-at-a-time type of sensitivity analysis. This type of sensitivity analysis is addressed as the simplest type of sensitivity analysis. ${ }^{21}$ Although in this type of analysis the influence of each independent variable can be clearly observed, the method does not reveal the effects on the dependent variables which may be caused by the concurrent change of the selected independent variables. ${ }^{22}$

The outcome of the repetitive simulations are summarized on figures 5 and 6 . Figure 5 represents the simulation results when the data retrieved from the leader are directly used in the guidance and control algorithms. Figure 6 summarizes the results of the second set of simulations, in which the leader data is first processed by the prediction algorithm given in section III-C, before the guidance and control algorithms use them. The dead-reckoning-based algorithm predicts the horizontal position of the leader aircraft based on its horizontal velocity and timestamp information, and makes its prediction available to the guidance algorithm at each iteration. The algorithm also uses the same velocity and timestamp information to compensate the delay contained within the position data.

The figures show the variation of the components of $\left|\overline{\boldsymbol{e}}_{r}\right|_{\max }$ with respect to different independent variables. Each figure shows the influence of two independent variables while the third independent variable is kept constant. In the left-side column of the figures 5 and 6 , the variation of the components of $\left|\overline{\boldsymbol{e}}_{r}\right|_{\max }$ with respect to the amplitude of noise and the level of transfer delay is shown. The right-side column of the figures 5 and 6 illustrate the effect of the noise amplitude and transfer period on the components of $\left|\overline{\boldsymbol{e}}_{r}\right|_{\text {max }}$. The curves are colored in such a way that a darker curve means a greater value of the corresponding independent variable, and vice versa. The figure legends show only the uppermost and lowermost values of the corresponding independent variables in order to save space.

\section{A. Discussion of Results With Direct Use of Leader Data}

Looking at different components of the relative position error $\left|\overline{\boldsymbol{e}}_{r}\right|_{\max }$ in figure 5 , it can be seen that the $\mathrm{x}$-component of the error shown in figures 5(a) and 5(b) is the most sensitive component of the error against the delay in the leader information and the increasing transfer period of the data. This is because, as the leader aircraft is flying straight and level, a significant component of the follower aircraft's velocity is pointing towards the leader aircraft. Hence the follower aircraft's velocity vector has its greatest component on the longitudinal (x) axis of the leader-fixed reference frame. Presence of delay in the leader aircraft data causes an increase in the x-component of the formation flight error, because the follower aircraft guidance and control algorithms work with past data of the leader aircraft. So if the leader aircraft position data contain i.e. 0.2 seconds of delay, recalling that the leader aircraft is flying with a speed of $35 \mathrm{~m} / \mathrm{s}$, the follower aircraft will follow the leader aircraft at a position $0.2 \times 37=7$ meters behind the desired relative position. This amount of error is visible on the figure $5(\mathrm{a})$ on the curve corresponding to $n=0$. In figure 5 (b) it can be seen that, the transfer period of the leader data is another important factor affecting the longitudinal relative position error during the formation flight. The formation control algorithms process the most recently-received leader aircraft data. If the leader aircraft data transfer period is greater than the data processing period of the follower aircraft, the formation guidance algorithms need to process past information of the leader aircraft data that it has most recently received. This occurs until new leader data is received, causing position error in a way similar to the effect of delay. The figures 5(a) and 5(b) also show that increasing level of noise also causes an expected increase on the amount of the position error. As the amplitude of noise in leader position and velocity data increases, the curves shift in the positive direction of the vertical axis with negligible change in its slope. This means that increasing noise amplitude in the leader position and velocity data adds a constant value on the $\left|\overline{\boldsymbol{e}}_{x}\right|_{\text {max }}$. This behavior is also present on lateral and vertical components of error shown in figures $5(\mathrm{c})-5(\mathrm{f})$.

In figures $5(\mathrm{c})$ and $5(\mathrm{~d})$ the effect of the independent variables on the maximum lateral (y) component of the formation keeping error can be observed. Figure 5(c) shows that increasing delay does not affect the lateral position keeping performance during the formation flight. This is due to the fact that the leader aircraft flies a straight path. So processing the outdated lateral position information of the leader aircraft does not add up to the relative lateral position error, since the follower aircraft's lateral velocity component is very small. A similar behavior is present in figure 5(b) for low values of $n$, as the independent variable, transfer period, is increased. However at higher values of the noise amplitude factor, a slight increase of $\left|\bar{e}_{y}\right|_{\max }$ becomes apparent with increasing transfer period. One factor affecting this behavior is that, at 


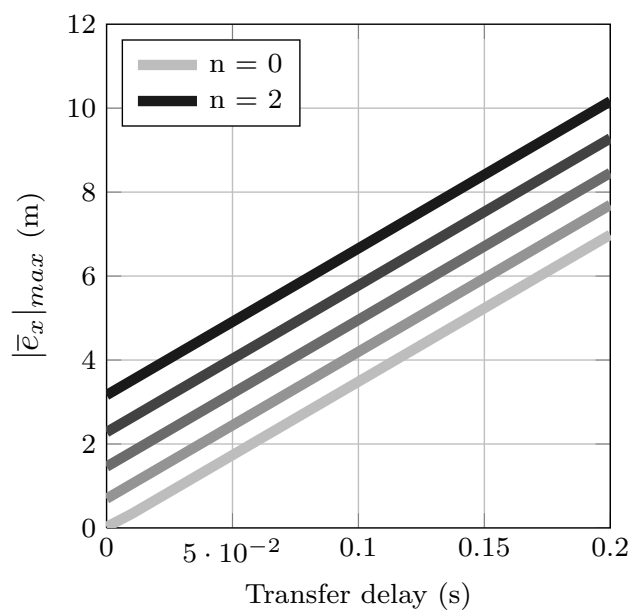

(a) Variation under constant transfer period of $0.02 \mathrm{~s}$

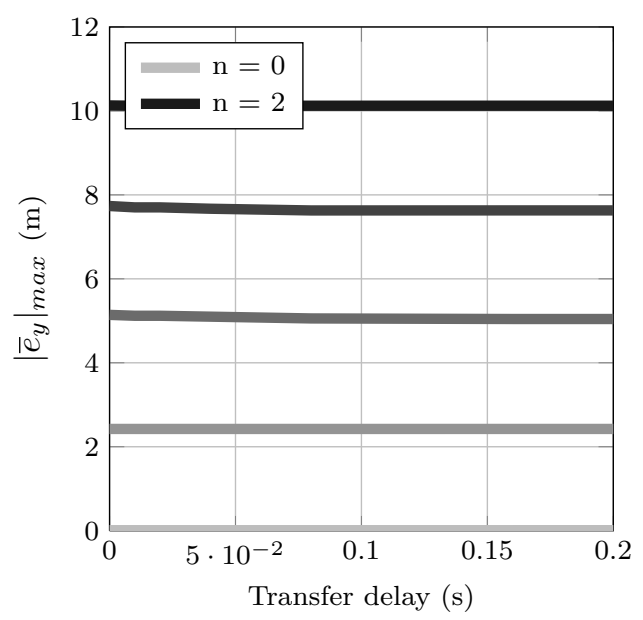

(c) Variation under constant transfer period of $0.02 \mathrm{~s}$

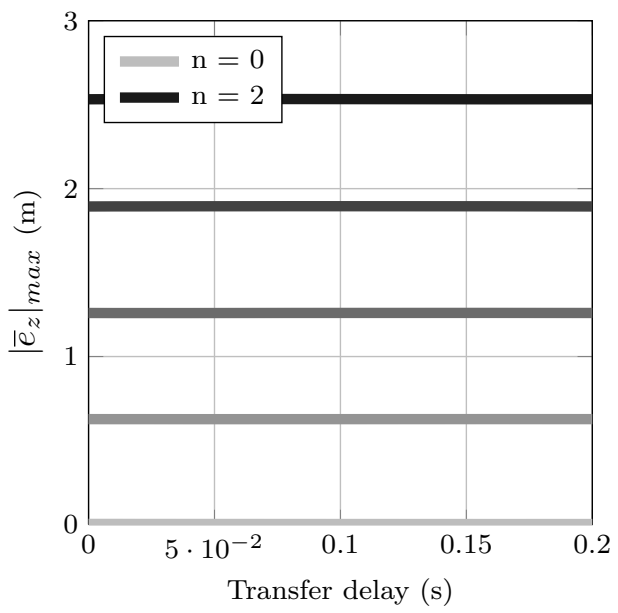

(e) Variation under constant transfer period of $0.02 \mathrm{~s}$

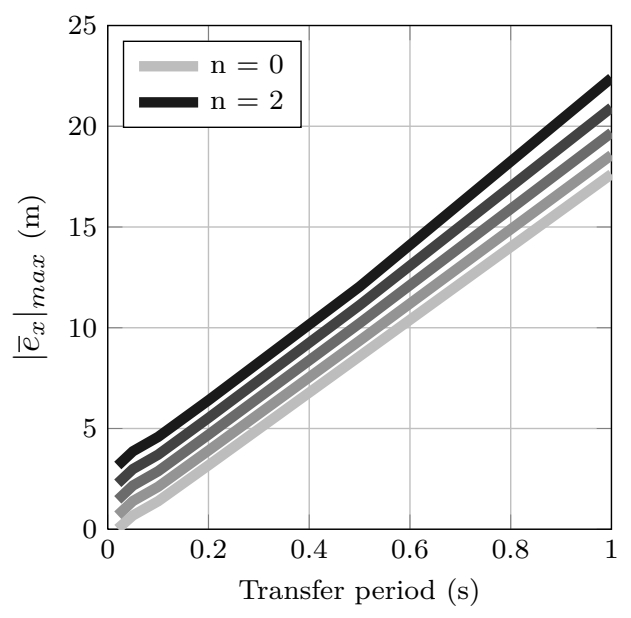

(b) Variation under constant transfer delay of $0 \mathrm{~s}$

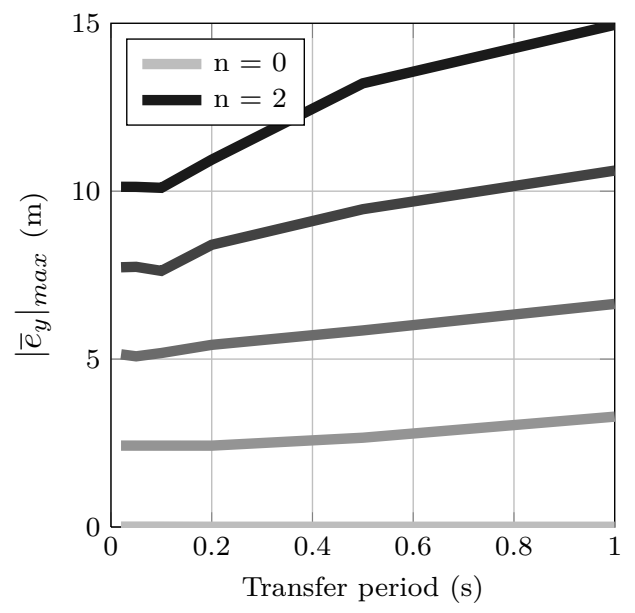

(d) Variation under constant transfer delay of $0 \mathrm{~s}$

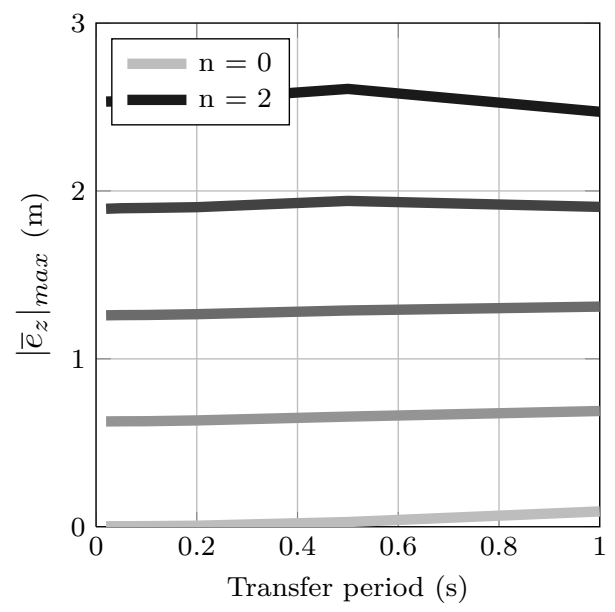

(f) Variation under constant transfer delay of $0 \mathrm{~s}$

Figure 5. Variation of maximum absolute error with independent variables. No filter is applied to the gathered leader data. 
different transfer periods, different instances of the noise-corrupted leader data is processed by the follower aircraft, whose noise values vary at different instants (see figure 3). Another factor is that, as the transfer period increases, the follower aircraft will have more time to react to the relative position command, in which position errors are present due to the noisy and less accurate flight data obtained from the leader aircraft.

Finally looking at figures 5(e) showing the influence of the independent variables on the vertical (z) component of the maximum relative position error $\left|\overline{\boldsymbol{e}}_{r}\right|_{\max }$, a behavior similar to that of the lateral error can be observed. Since the flight path of the leader aircraft is a level path, the non-time-varying altitude of the leader aircraft does not cause the vertical error to increase whether the follower aircraft process the outdated or the current information of the leader aircraft. However it is shown on figure 5(f) that, the $\left|\bar{e}_{z}\right|_{\max }$ remains constant with increasing transfer period, unlike the $\left|\bar{e}_{y}\right|_{\text {max }}$ shown in $5(\mathrm{~d})$. The different smoothness characteristics and local extrema of the added noise data on lateral and vertical channels could be a reason for obtaining different behaviors. Another reason is likely to be how the data are processed by the formation guidance algorithms. On the lateral channel of the formation guidance algorithm shown in equation 3, two sources of the leader information are used: the course angle $\chi_{L}$ and position. While on the vertical channel only the position information of the leader aircraft is used. Therefore on the lateral channel there are two sources, namely the course angle and position, where the noise in the leader flight data enter into the control loop.

\section{B. Discussion of Results With the Use of Predicting Filter}

Figure 6 shows the results of the simulations, in which the gathered leader aircraft data is processed by a predicting filter before being processed by the guidance algorithms. The predicting filter provides a velocitybased position estimation at each iteration of the guidance and control algorithms, exemplifying a possible way of reducing formation keeping error caused by the delay and high data transfer period. As figures 6(a) and $6(\mathrm{~b})$ are compared with figures $5(\mathrm{a})$ and 5(b), it can be seen that using the leader prediction algorithm reduces dependence of the error magnitude on delay and transfer period. This is because the prediction algorithm provides an estimate of the current leader position, so the guidance algorithm does not have to process past data of the leader aircraft. However at higher values of $n$, the accuracy of the position estimates of the prediction algorithm deteriorates, as at higher values of $n$, the velocity information becomes less accurate, on which the position estimates are based. Therefore the effectiveness of the prediction algorithm is limited to low amplitude of noise on the leader velocity data.

It is shown in figure 5(c) that the lateral component of the formation keeping error is not affected by the delay contained within the leader aircraft flight data, due to the straight and level path of the leader aircraft. Since the prediction filter is used to compensate for the delay and high transfer period of the leader data, the filter is not expected to make improvement where the delay and the transfer period are already not affecting the error components. Comparison of the figures 5(c) and 6(c) show that this is indeed the case. The lateral component of the error remain unaffected from the delay variations. However, comparing figures $5(\mathrm{~d})$ and $6(\mathrm{~d})$ it can be seen that the filter amplifies the position error at high values of $n$ due to the low accuracy of the velocity data. Based on that, it can be said that the application of such a filter for compensation of high transfer period effects are only useful, if the correction data (here the leader velocity) is considerably accurate.

Since the predicting filter only provides the horizontal position estimates, no significant change is present in figures $6(\mathrm{e})$ and $6(\mathrm{f})$ relative to the figures $5(\mathrm{e})$ and $5(\mathrm{f})$, where the vertical component of $\left|\overline{\boldsymbol{e}}_{r}\right|_{\text {max }}$ is shown.

\section{Conclusion}

Using a simulation-based sensitivity analysis, the effect of noise level, the delay and the transfer period of the leader position and velocity data on the formation keeping performance is presented. A simple deadreckoning algorithm is also included in the simulations in order to represent the case of filtering the leader data before sending them to the formation guidance algorithms. For the case of directly using the leader aircraft data on the follower aircraft, the simulations show that all of the parameters have considerable effects on the performance of the formation flight. The greatest influence of the delay and transfer period is observed on the longitudinal component of the relative position error, along which the follower aircraft has the greatest portion of its velocity, as the formation keeping error build up in this direction proportional to the velocity component and corresponding delay or transfer period of the leader data. Simulations with 


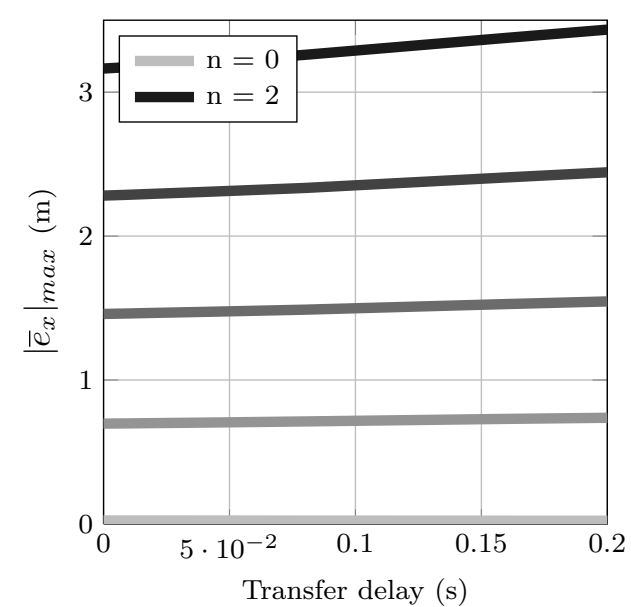

(a) Variation under constant transfer period of $0.02 \mathrm{~s}$

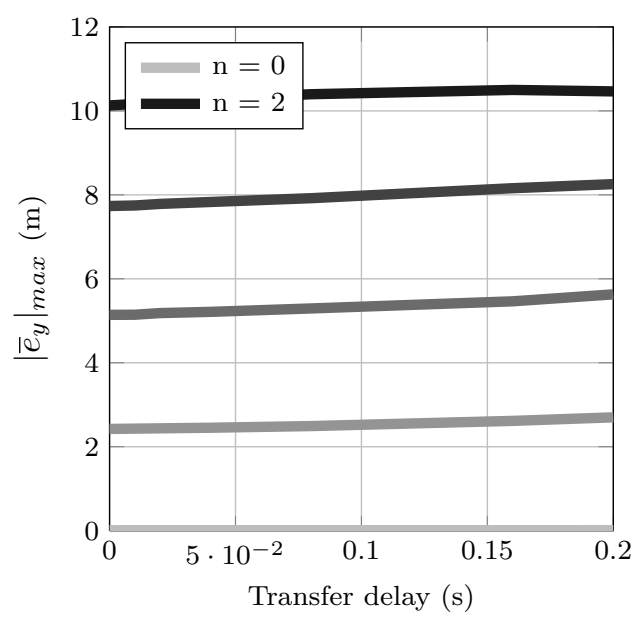

(c) Variation under constant transfer period of $0.02 \mathrm{~s}$

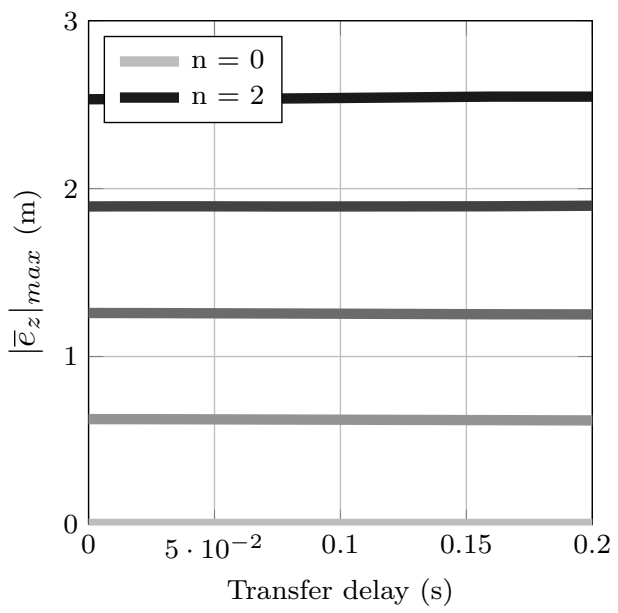

(e) Variation under constant transfer period of $0.02 \mathrm{~s}$

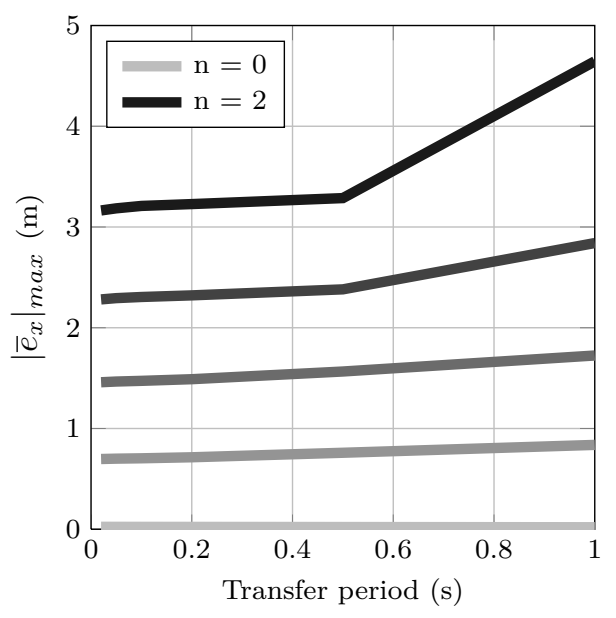

(b) Variation under constant transfer delay of $0 \mathrm{~s}$

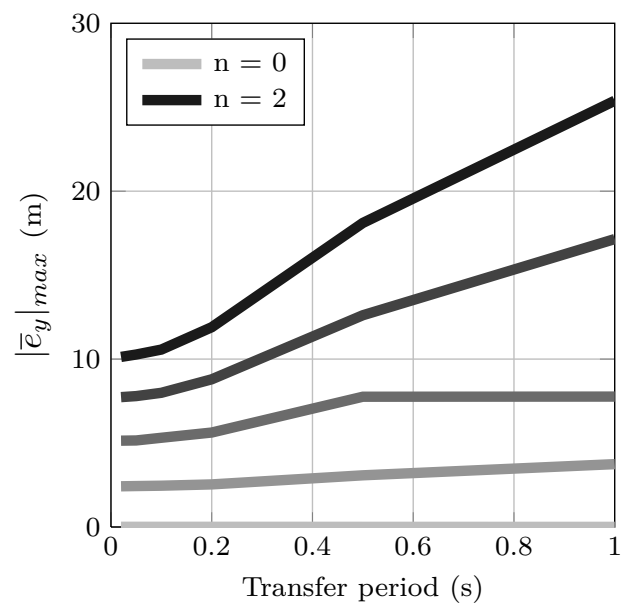

(d) Variation under constant transfer delay of $0 \mathrm{~s}$

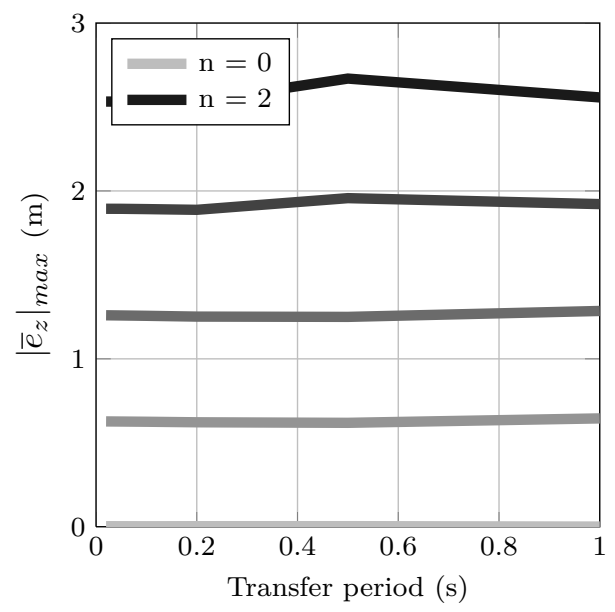

(f) Variation under constant transfer delay of $0 \mathrm{~s}$

Figure 6. Variation of maximum absolute error with independent variables. A dead-reckoning-based filter (section III-C) is applied to the leader data for compensating delay and predicting leader states. 
the dead-reckoning filter showed that the negative effects associated with the delay and the transfer period can be reduced to an extent that the accuracy of the leader velocity information permits. However such a filter may also amplify the noise present in the position data, in case non-accurate information is used for position prediction. Therefore for the studied formation flight application, the most critical parameter of the data gathering method is regarded as the accuracy of the leader aircraft information. The other parameters, namely the delay and the transfer rate characteristics of the data gathering method are regarded as of secondary importance. This is because, only leader data of sufficient accuracy allow a simple prediction algorithm to provide effective delay and transfer period compensation.

\section{Acknowledgements}

The authors would like to thank Sven Lorenz and Johann C. Dauer for their helpful suggestions. This research has been funded by the company Airbus Defence \& Space, under its project Sagitta.

\section{References}

${ }^{1}$ Okolo, W., Dogan, A., and Blake, W., "Application of Sweet Spot Determination to a Conventional Pair of Aircraft," AIAA Atmospheric Flight Mechanics Conference, Minneapolis, Minnesota, 13-16 August 2012.

${ }^{2}$ Osteroos, R. K., Ferris, D. L., Maddocks, J. R., Moreno, I. P., and Sabella, M., "Limited Evaluation of the Formation Flight Controller (Project Solo Form)," AFFTC-TIM-04-08, Air Force Flight Test Center, Edwards Air Force Base, California, Dec. 2002.

${ }^{3}$ Dibley, R. P., Allen, M. J., and Nabaa, N., "Autonomous Airborne Refueling Demonstration Phase I Flight-Test Results," AIAA Atmospheric Flight Mechanics Conference and Exhibit, Hilton Head, South Carolina, 20-23 August 2007.

${ }^{4}$ Hansen, J., Romrell, G., Nabaa, N., Andersen, R., Myers, L., and McCormick, J., "DARPA Autonomous Airborne Refueling Demonstration Program with Initial Results," ION GNSS 19th International Technical Meeting of The Satellite Division, Fort Worth, Texas, 26-29 September 2006.

${ }^{5}$ Pachter, M., D'Azzo, J. J., and Proud, A. W., "Tight Formation Flight Control," Journal of Guidance, Control, and Dynamics, Vol. 24, No. 2, 2001.

${ }^{6}$ Hanson, C. E., Ryan, J., Allen, M. J., and Jacobson, S. R., "An Overview of Flight Test Results for a Formation Flight Autopilot," NASA/TM-2002-210729, NASA Dryden Flight Research Center, Edwards, California, Aug. 2002.

${ }^{7}$ Osteroos, R. K., Pachter, M., and Jacques, D. R., "Full Capability Formation Flight Control," AIAA Guidance, Navigation and Control Conference and Exhibit, 2004.

${ }^{8}$ Campa, G., Napolitano, M. R., Seanor, B., and Perhinschi, M. G., "Design of Control Laws for Maneuvered Formation Flight," American Control Conference, 2004.

${ }^{9} \mathrm{Gu}$, Y., Seanor, B., Campa, G., Napolitano, M. R., and Rowe, L., "Design and Flight Testing Evaluation of Formation Control Laws," IEEE Transactions on Control Systems Technology, Vol. 14, No. 6, 2006, pp. 1105-1112.

${ }^{10}$ Ross, S. M., Pachter, M., Jacques, D. R., Kish, B. A., and Millman, D. R., "Autonomous Aerial Refueling Based on the Tanker Reference Frame," Aerospace Conference, IEEE, 2006.

${ }^{11}$ You, D. I. and Shim, D. H., "Autonomous Formation Flight Test of Multi-Micro Aerial Vehicles," Journal of Intelligent Robotic Systems, Vol. 61, No. 1-4, Jan. 2011, pp. 321-337.

${ }^{12}$ Segal, S., Ben-Asher, J. Z., and Weiss, H., "Derivation of Formation Flight Guidance Laws for Unmanned Air Vehicles," Journal of Guidance, Control, and Dynamics, Vol. 28, No. 4, 2005.

${ }^{13}$ Tahk, M.-J., Park, C.-S., and Ryoo, C.-K., "Line-of-Sight Guidance Laws for Formation Flight," Journal of Guidance, Control, and Dynamics, Vol. 28, No. 4, 2005.

${ }^{14}$ Wilson, D. B. and Goktogan, A. H., "An Unmanned Aerial Vehicle Rendezvous and Formation Flight Demonstration," International Conference on Unmanned Aircraft Systems (ICUAS), Philadelphia, 12-15 June 2012.

${ }^{15}$ Venkataramanan, S. and Dogan, A., "A Multi-UAV Simulation for Formation Reconfiguration," AIAA Modeling and Simulation Technologies Conference and Exhibit, Providence, Rhode Island, 16-19 August 2004.

${ }^{16}$ Johnson, E. N., Calise, A. J., Watanabe, Y., Ha, J., and Neidhoefer, J. C., "Real-Time Vision-Based Relative Aircraft Navigation," Journal of Aerospace Computing, Information, and Communication, Vol. 4, 2007.

${ }^{17}$ Kilic, M. C. and Dauer, J. C., "Robustness Analysis of a Formation Flight Guidance Algorithm for Automated Aerial Refueling," AIAA Guidance, Navigation and Control Conference, Boston, Massachusetts, 19-22 August 2013.

${ }^{18}$ Sharif, M., Stein, A., and Schetselaar, M., "Integrated approach to predict confidence of GPS measurement," 20th ISPRS Congress, Istanbul, Turkey, 12-23 July 2004.

${ }^{19}$ Weltzien, C., "GPS Empfänger Vergleich," DLG-Prüfbericht-5148f, Deutsche Landwirtschafts-Gesellschaft e.V., GrossUmstadt, Germany, March 2003, in German.

${ }^{20}$ Bever, G., Urschel, P., and Hanson, C. E., "Comparison of Relative Navigation Solutions Applied Between Two Aircraft," NASA/TM-2002-210728, NASA Dryden Flight Research Center, Edwards, California, March 2002.

${ }^{21}$ Hamby, D. M., "A Review of Techniques for Parameter Sensitivity Analysis of Environmental Models," Environmental Monitoring and Assessment, Vol. 32, 1994, pp. 135-1542.

${ }^{22}$ Czitrom, V., "One-Factor-at-a-Time Versus Designed Experiments," The American Statistician, Vol. 53, No. 2, May 1999. 